\title{
Abkürzungen
}

Im Astronomischen Jahresbericht sind die unter I-V angeführten Abkür. zungen zur Anwendung gekommen.

\section{Allgemeine Abkürzungen}

$\begin{array}{ll}\text { Abh. } & \text { Abhandlungen } \\ \text { Abstr. } & \text { Abstrakt, abstract } \\ \text { Acad. } & \text { Académie, Academy } \\ \text { Accad. } & \text { Accadémia } \\ \text { Akad. } & \text { Akademie } \\ \text { Ann. } & \text { Annalen, Annales, Annals } \\ \text { Astr. } & \text { Astronomie etc. } \\ \text { Astrophys. } & \text { Astrophysik etc. } \\ \text { Beob. } & \text { Beobachtungen } \\ \text { Ber. } & \text { Berichte } \\ \text { Bol. } & \text { Boletin } \\ \text { Bull. } & \text { Bulletin } \\ \text { bzw. } & \text { beziehungsweise } \\ \text { Circ. } & \text { Circular, Zirkular } \\ \text { Cl. } & \text { Classe } \\ \text { Commun. } & \text { Communications } \\ \text { Comun. } & \text { Comunicazioni } \\ \text { Contr. } & \text { Contributions etc. } \\ \text { CR. } & \text { Comptes Rendus } \\ \text { Desgl. } & \text { Desgleichen } \\ \text { Diss. } & \text { Dissertation } \\ \text { Geod. } & \text { Geodäsie etc. } \\ \text { Ges. } & \text { Gesellschaft } \\ \text { Inst. } & \text { Institut etc. } \\ \text { J. } & \text { Journal } \\ \text { Kl. } & \text { Klasse } \\ \text { Mag. } & \text { Magazine } \\ \text { Math. } & \text { Mathematik etc. } \\ \text { Medd. } & \text { Meddelanden, } \\ & \text { Meddelelser } \\ \text { Mém. } & \text { Mémoires } \\ \text { Mem. } & \text { Memoirs, Memorie } \\ \text { Mitt. } & \text { Mitteilungen } \\ \text { Nachr. } & \text { Nachrichten } \\ \text { (NF) } & \text { Neue Folge } \\ \text { (NR) } & \text { Neue Reihe } \\ & \end{array}$

(NS) Neue Serie

Obs. Observatorinm

Oss. Osservatorio,

Phil Osservazion

Phys. $\quad$ Physik etc.

Proc. Proceedings

Pubbl. Pubblicazioni

Publ. Publikationen, Publi-

Rech. Recherches

Ref. Referat

Rend. Rendiconti

Rep. Report

Repr. Reprint

Res. Research

Rev. Review, Revue

Ric. Ricerche

Sci. Science, scientific

Ser. Serie, Series

Sér. Série

Sitzber. Sitzungsberichte

Soc. Società, Société, Society

Sternw. Sternwarte

Suppl. Supplement

T.

Tidskrift, Tidsskrift, Tijdschrift

Trans. Transactions

Univ. Universität etc.

Veröff. Veröffentlichungen

Vgl. Vergleiche

VJS Vierteljahrsschrift

Wetensch. Wetenschappen

Wiss. Wissenschaften

Z. Zeitschrift 


\section{Spezielle Abkürzungen}

Die nachstehenden Abkürzungen sind insbesondere in $\S 75$ Nachweis der Beobachtungen und Berechnungen Kleiner Planeten, ferner in $\S 81$ Einzelne Kometen, § 112 Veränderliche Sterne und in $\$ 113$ Novae, Supernovae verwendet.

$\begin{array}{ll}\text { aD } & \text { absolute Dimensionen } \\ \text { Am } & \text { Amplitude } \\ \text { An } & \text { Anzeige } \\ \text { Au } & \text { Aufnahme } \\ \text { B } & \text { Beobachtung } \\ \text { C } & \text { Farbe } \\ \text { CI } & \text { Farbenindex } \\ \text { E } & \text { Lichtwechselelemente } \\ \text { Eb } & \text { Photometrische Bahnelemente } \\ \text { EB } & \text { Eigenbewegung } \\ \text { El } & \text { Bahnelemente } \\ \text { Eph } & \text { Ephemeride } \\ \text { lis } & \text { Spektroskopische Elemente } \\ \text { Fsb } & \text { SpektroskopischeBahnelemente } \\ \text { H } & \text { Helligkeit bzw. physische Be- } \\ & \text { obachtung }\end{array}$

AAVSO . . . . . vgl. Ref. 1102 Ref. AAS . . . . vgl. Ref. 1104 Ref. AG . . . . . vgl. Ref. 1110

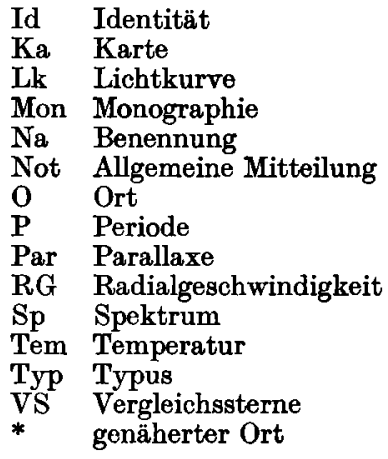

Ref. ASP . . . . vgl. Ref. 1107 Ref. RAS . . . vgl. Ref. 1122 Ref. Soc. Astr. Italiana vgl. Ref. 1127

\section{Abkürzungen von Ortsnamen (Sternwarten)}

Die Tabelle für die Abkürzungen der Ortsnamen ist auf Seite 242 im Nachweeis der Beobachtungen und Berechnungen Kleiner Planeten gegeben.

\section{Abkürzungen der Sternbild-Namen}

Für die Namen der Sternbilder sind die in Harvard Bulletin 771 veröffentlichten Abkürzungen benutzt. 


\section{Abkürzungen von Zeitschriften}

Die Nummern beziehen sich auf den ausführlichen Titel der Zeitschriften und Veröffentlichungen S. XIII und S. XX.

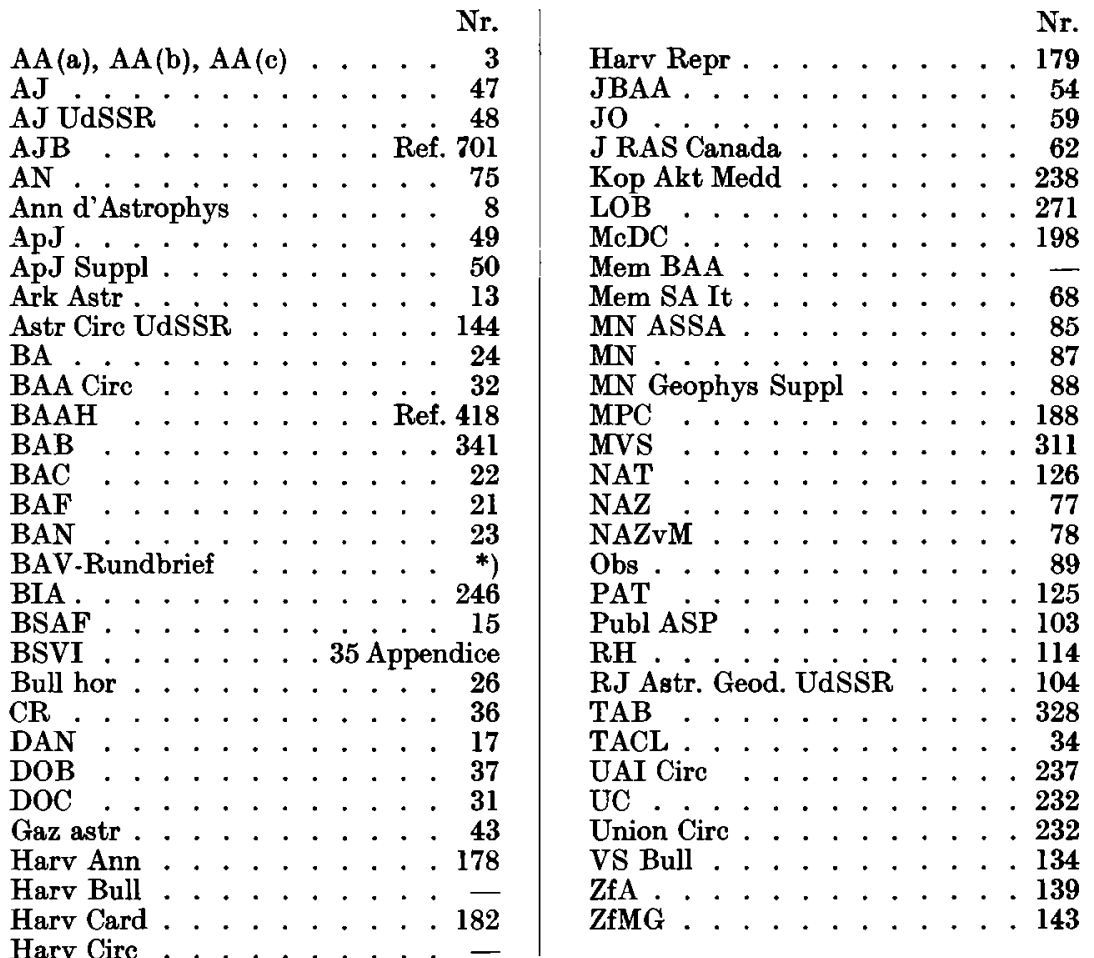

*) Mitteilungsblatt der Berliner Arbeitsgemeinschaft für Veränderliche Sterne.

\section{Zeitschriften}

Unter Zeitschriften sind nicht nur alle periodisch erscheinenden Schriften verstanden, sondern im weiteren Sinne auch alle zwanglos erscheinenden Veröfentlichungen von Sternwarten, Instituten, Gesellschaften und Akademien.

Die vollständig durchgesehenen Zeitschriften sind in den beiden folgenden Verzeichnissen VI und VII zusammengestellt.

Einzelne Zeitschriften, die nicht direkt eingesehen werden konnten, sind dadurch kenntlich gemacht, daß in den beiden Verzeichnissen ihre laufende Nummer eingeklammert ist.

Nicht aufgeführt sind in den Verzeichnissen VI und VII diejenigen Zeitschriften, in denen gelegentlich astronomische Arbeiten enthalten sind. Diese sind der Schriftleitung entweder nur in Form eines Sonderdruckes, als einzelne Abhandlung einer Akzademie oder als sonstige bibliographische Mitteilung bekannt geworden. 